\title{
The Anti-brain Ageing Effects of Krill Phosphatidylserine in SAMP10 Mice
}

\author{
Jun Wang ${ }^{\text {la }}$, Hongtao Lei ${ }^{2 \mathrm{a}}$, Pengtao $\mathrm{Li}^{3}$, $\mathrm{Li} \mathrm{Han}^{4}$, Jincai Hou ${ }^{5}$, Yangyang $\mathrm{Yan}^{3}$, Haiping Zhao ${ }^{6} \&$ Tomoko Tsuji ${ }^{4}$ \\ ${ }^{1}$ Institute of Basic Theory, China Academy of Chinese Medical Sciences, Dong Cheng District, Beijing, \\ China \\ ${ }^{2}$ The Experimental Research Center, China Academy of Chinese Medical Sciences, Dong Cheng District, \\ Beijing, China \\ ${ }^{3}$ Dongzhimen Hospital Affiliated to Beijing University of Chinese Medicine, Dongcheng District, Beijing, \\ China \\ ${ }^{4}$ Human Life Science R \& D Center, Nippon Suisan Kaisha, Ltd., 2-6-2 Otemachi, Chiyoda-ku, Tokyo, \\ Japan \\ ${ }^{5}$ Xiyuan Hospital of China Academy of Chinese Medical Sciences, Hai Dian District, Beijing, China \\ ${ }^{6}$ Cerebrovascular Diseases Research Institute, Xuanwu Hospital of Capital Medical University, Xin Cheng \\ District, Beijing, China
}

${ }^{a}$ These authors contributed equally to this paper

Correspondence: Pengtao Li, Dongzhimen Hospital Affiliated to Beijing University of Chinese Medicine, 5 Hai Yun Cang, Dongcheng District, Beijing 100700, China. Tel: 86-10-8401-3206. E-mail: lipengtao0413@hotmail.com

Received: May 22, 2012 Accepted: June 6, 2012 Online Published: August 8, 2012

doi:10.5539/jas.v4n9p196 URL: http://dx.doi.org/10.5539/jas.v4n9p196

\begin{abstract}
We examined the biological effect of krill phosphatidylserine (K-PS) on brain ageing and investigated the mechanisms that how K-PS works on the brain using senescence-accelerated mouse (SAM) model with age-associated neurodegeneration. SAMP10 mice with 5 months of age were treated orally once a day for 75 days with 10 or $100 \mathrm{mg} \mathrm{kg}^{-1} \mathrm{~d}^{-1} \mathrm{~K}$-PS (low and high dose). The effect of K-PS treatment on the cross-sectional area and Nissl body number of the neocortex at point $C$, an area prone to atrophy in SAMP10 mice, behavior and oxidative stress were evaluated by Image-Pro Plus software, step-down testing, and malondialdehyde (MDA) and glutathione peroxidase (GSH-Px) assays. Ionized calcium binding adaptor molecule 1 (Iba-1) and insulin-like growth factor 1 (IGF-1) expression was evaluated using immunohistochemistry. Low or high doses of K-PS significantly increased the cross-sectional area and Nissl body number at point $C$, partly reversed memory impairment, increased the activity of GSH-Px and reduced MDA levels. Moreover, immunohistochemistry indicated that K-PS suppressed Iba-1 expression and upregulate the expression of IGF-1. These findings suggest that K-PS could prevent or slow the progression of brain atrophy and neuronal damage associated with aging by the inhibition of Iba-1 and the upregulation of IGF-1 expression.
\end{abstract}

Keywords: krill phosphatidylserine, SAMP10, ageing, neurodegeneration

\section{Introduction}

Ageing, which is characterized by extensive degeneration in terms of morphology and function in the brain tissue, has been an important risk factor for the kinds of neurodegenerative diseases such as Alzheimer's disease (AD) and Parkinson's disease (Stern et al., 1987; Prasad \& Aggarwal, 2011). With older age, there is loss of neuronal cells in neocortical, hippocampal, and cerebellar areas, shrinkage of neurons, leading to compromised neuronal integrity and reduction in synaptic density (Simić et al., 1997; Morrison \& Hof, 2007). Consequently, age is believed to increase risk for AD because it is independently linked to brain atrophy (Raz et al., 2004). 
Brain tissue atrophy means the reduction of neurons and the loss of neuron-neuron connections. Atrophy generates either widespread, which means a shrunk of whole brain tissue; or locally which means a damage of limited region of the brain.

Phosphatidylserine (PS) is a member of natural phospholipids and an essential cell membrane building-block. It has been known that PS is one of the important brain phospholipids and plays a key role in maintaining the structure and function of normal neurons. PS and other essential fatty acids especially the docosahexaenoic acid (DHA), may also play an important role in many functions of neuron membranes, such as release of neurotransmitters, signal transduction, cell's communication, cell growth and apoptosis (Vance \& Steenbergen, 2005). On the other hand, the report about association between ageing and alterations in brain lipid composition (Svennerholm et al., 1991), suggested that phospholipids, as the fundamental components of neuronal membranes, may serve as effective treatment for cognitive deterioration.

The first generation PS material was the PS extracted from bovine cortex (BC-PS). Administration of BC-PS to patients suffered from age-associated memory damage could significantly improve the performance of attention, learning and memory (Crook et al., 1991). Lots of other studies have also shown that treatment with PS can counteract the pathological dysfunctions in patients with age-related disorders and improve the ability of memory and learning in rats (Corwin et al., 1985; Delwaide et al., 1986; Zanotti et al., 1986; Amaducci, 1988). However, to avoid the possibility of the contamination by protein prions of bovine spongiform encephalopathy (BSE), BC-PS was limited in use, and more safety alternative material was needed. As the second generation PS, soybean PS (SB-PS) was developed by phospholipase D-catalyzed transphosphatidylation of soybean phosphatidylcholine (also known as soy lecithin). However, there still lack of evidence to support this use.

Surely, preliminary studies in rats indicate that SB-PS may have comparable effects on cognition when compared with BC-PS (Blokland et al., 1999). But, later clinical trials proved that a daily administration of SB-PS does not change memory or other cognitive functions in older patients with memory dysfunction (Jorissen et al., 2001). In fact, the fatty acids attached to the phospholipids in the SB-PS are not identical to those in the BC-PS, because the high unsaturated fatty acids (HUFA), especially DHA hardly existing in fatty acid of soybean. It is surmised that this fault caused that above-mentioned results were not consistent. Therefore, development of a new safety and powerful PS material is expected.

Recently, the marine PS which extracted from marine life, such as krill, squid and fish, was expected to become the third generation of PS, and has been regarded as a hopeful alternative of BS-PS because of the substantial amount of omega-3 HUFA, especially DHA and eicosapentaenoic acid (EPA) in a phospholipid form. A recent study showed that krill PS (K-PS), which transphophatidylated from krill phosphatidylcholine (K-PC), ameliorated the spatial memory ability and protected against cholinergic cell injure in aged rats (Lee et al., 2010). In a double-blind placebo-controlled trial, the result indicated that supplementation of $300 \mathrm{mg} /$ day PS-DHA for 15 weeks may recover the degenerative memory in elderly without dementia (Vakhapova et al., 2010).

The senescence-accelerated mouse (SAM) has been well documented in the historical development of animal models of ageing (Shimada et al., 1992 \& 2003). A lot of studies by SAM model have been conducted in different region of ageing science, and SAMP10 mice, as a distinctive model of age-related, inherited cerebral atrophy with cognitive dysfunction, have been reviewed extensively (Shimada, 1999). Although there is evidence that K-PS produce neuroprotective activity, no detailed and further study of its effect on neurodegeneration using SAMP10 mice has been reported to date to our knowledge. In the study reported here, SAMP10 mice were selected as a model of neurodegeneratipn to explore whether treatment with K-PS can reduce neuronal damage and behavioral deficits, and to investigate the mechanisms by which K-PS affects the brain.

\section{Materials and Methods}

\subsection{Mice}

Five-month-old of male SAMP10 and SAMR1 strains of mice were purchased from the First Teaching Hospital of Tianjin University of Traditional Chinese Medicine (SCXK2008-0001, No.0001375, Tianjin, China). The mice were kept in a room under a controlled temperature of $23 \pm 2^{\circ} \mathrm{C}$, humidity of $50 \pm 10 \%$ and a 12-hour light-dark cycle during study periods. Certified commercial rodent diet (Beijing Hua Fu Kang Laboratories) and sterile water were provided ad libitum. The SAMP10 mouse was used as a model of age-related cerebral degeneration, while the SAMR1 mouse was used as a control strain for normal ageing. The animal experiments 
were carried out according to the UK Animals (Scientific Procedures) Act 1986 and concerned guidelines for the care and use of laboratory animals at our institution.

\subsection{Materials and Regents}

$\mathrm{K}-\mathrm{PS}$, as a test sample, and medium chain triglyceride as a placebo sample or to dilute test samples, were obtained from Nippon Suisan Kaisha, Ltd. (Tokyo, Japan). K-PS was made by phospholipase D-catalyzed transphophatidylation of krill phosphatidylcholine (K-PC), which was extracted with ethyl alcohol from Antarctic krill (Euphausia superba Dana). It contained $43.9 \%$ phospholipids, $43.4 \%$ triglyceride, $11.3 \%$ EPA and $5.6 \%$ DHA by weight (wt \%). A great portion of EPA and DHA are combined with phospholipids (Table 1).

Table 1. Fatty acid composition of K-PS and MCT (\%)

\begin{tabular}{lccccccccccccccc}
\hline & C8:0 & C10:0 & C14:0 & C16:0 & Cl6:1 & C18:0 & C18:1 & C18:2 & C18:3 & C18:4 & C20:4 & C20:5 & C22.5 & C22:6 & 0 thers \\
\hline K.PS Total & & & 11.9 & 21.6 & 6.1 & 1.3 & 17.7 & 1.7 & 1.2 & 3.4 & 0.3 & 15.2 & 0.4 & 7.7 & 11.5 \\
$\quad$ PL Form & & & 3.4 & 34.5 & 1.7 & 1.5 & 12.6 & 1.7 & 1.3 & 1.6 & 0.3 & 21.7 & 0.5 & 10.1 & 9.1 \\
$\quad$ TG Form & & & 17.8 & 20.1 & 9.0 & 1.4 & 22.7 & 1.8 & 1.1 & 4.0 & & 5.2 & 0.2 & 2.3 & 14.4 \\
\hline MCT & 58.3 & 41.3 & & & & & & & & & & & & & 0.4 \\
\hline
\end{tabular}

C8:0 = Caprylic acid; C10:0 = Capric acid; C12:0 = Lauric acid; C14:0 = Myristic acid; C16:0 = Palmitic acid; C16:1 = Palmitoleic acid; C18:0 = Stearic acid; C18:1 = Oleic acid; C18:2 = Linoleic acid; C18:3 = Linolenic acid; C18:4 = Cis-6, cis-9, cis-12, cis-15-octadecatetraenoic acid; C20:4 = Arachdonoic acid (AA); C20:5 = Ecosapentaenoic acid (EPA); C22:5 = Cis-4, cis-7, cis-10, cis-13, cis-16-docosapentaenoic acid (DPA); C22:6 = Docosahexaenoic acid (DHA).

For the experiment, K-PS low $\left(10 \mathrm{mg} \mathrm{kg}^{-1} \mathrm{~d}^{-1}\right)$ and K-PS ${ }_{\text {high }}\left(100 \mathrm{mg} \mathrm{kg}^{-1} \mathrm{~d}^{-1}\right)$ were administrated to the 5-month-old SAMP10 mice, and the vehicle (MCT) was administrated to the 5-month-old SAMR1 mice orally one a day for 75 days. The terminal month-age of mice was seven and a half month age which was regarded as aged SAM mice. Untreated 5-month-old SAMP10 and SAMR1 mice were selected as the baseline groups.

Malondialdehyde (MDA) and glutathione peroxidase (GSH-Px) kits were purchased from Beyotime Institute of Biotechnology, China (S0107, S0131, S0056). Anti-Iba-1 antibody was purchased from Abcam, United Kingdom (ab5076). Anti-IGF-1 antibody was purchased from Santa Cruz Biotechnology, USA (H-70). Other chemicals in this study were analytical grade or of the highest grade available which were purchased from Sigma Chemical Co. (St. Louis, MO)

\subsection{Microscopic Morphometry and Cortical Cross-sectional Area}

SAMP10 and SAMR1 mice were anesthetized with $10 \%$ chloral hydrate $\left(0.4 \mathrm{ml} \mathrm{kg}^{-1}\right.$ i.p.). The brains were rapidly removed and immersed in $10 \%$ neutral buffered formalin for 2 weeks. Brain tissues were serially sectioned into $100-\mu \mathrm{m}$-thick coronal slices from the olfactory bulbs to reach the landmark point $\mathrm{C}$, which is the most prominent degree of atrophy in SAMP10 mice (Shimada et al., 1994). From this, we countered the cross-sectional area of the neocortex at point $\mathrm{C}$ using Image-Pro Plus software (version 4.0, Media Cybernetics, Bethesda, MD).

\subsection{Histology}

For the Nissl body staining in the cross-sectional area of the neocortex at point $\mathrm{C}$, mice were perfused with saline transcardially, and then fixed with $4 \%$ paraformaldehyde solution. After dehydrated, the brain tissues were cut into transparent slices and imbedded in paraffin. Coronal sections of $5-\mu \mathrm{m}$ thick were stained with $0.5 \%$ thionien for $10 \mathrm{~min}$ at a temperature of $50^{\circ} \mathrm{C}$. Graded alcohol were used for dehydration and then the mounted with neutral balsam medium to observe changes of cross-sectional area at point C. Nissl body number was determined by counting the positive staining number with an inverted microscope at $400 \times$. Six visual fields were chosen randomly for each group. 


\subsection{Step-down Test}

A step-down passive avoidance computer-aided controlling system (YLS-3TB, Gene\&I, Beijing, China) was conducted to show the changes between SAMP10 and SAMR1 groups in their learning and memory. The equipment and test procedure were similar to those described by Shuchang et al. (2008). All mice were tested using a step-down test in a passive avoidance acrylic chamber. The unit consisted of copper rods and a well-insulated $4 \mathrm{~cm} \times 4 \mathrm{~cm} \times 5 \mathrm{~cm}$ platform made of rubber in one corner of the chamber. The mice were placed in the chamber for a 3-minute adaptation period and then placed the mice on the platform. Their latency to step down on the grid with all four paws was measured. Once the mice stepped down on the copper bars, they would receive a $0.2-\mathrm{mA}$ scrambled foot shock. The animals showed an instinctive reaction to jump back onto the platform to avoid the electric shock. Mice were tested in this manner for 5 minutes. The number of times that the mice stepped down from the platform within the 5-minute period was recorded as acquisition errors. Twenty-four hours later, this same procedure was repeated, and the step-down meaning of latency time was used as a measure of memory retention. The number of times that the mice stepped down onto the platform within the 5-minute interval was recorded as retention errors. All behavioral testing procedures were conducted in a blinded environment.

\subsection{Evaluation of GSH-Px and MDA}

Four to six mice were used for each group. Brains were rapidly moved into ice-cold phosphate buffered saline (PBS) and scraped in lysis buffer. The insoluble material was centrifuged at 12,000 rpm for $20 \mathrm{~min}$ and the supernatants were used for the determination of GSH-Px activities and MDA levels. GSH-Px activity was measured by the method of Lawrence and Burk (Lawrence \& Burk, 1976) with modifications. The absorbance was recorded at $340 \mathrm{~nm}$, immediately and then every $30 \mathrm{sec}$ over a period of $3 \mathrm{~min}$, using a Spectronic-20 spectrophotometer. GSH-Px activity was expressed as micro-unit per $\mathrm{mg}$ of fresh weight. Level of lipid peroxidation was expressed as the content of malondialdehyde (MDA) according to Zhang et al. (2005). The procedure was conducted with MDA commercial kits according to the manufacturer's instructions. The absorbance of the supernatant was recorded at $532 \mathrm{~nm}$ (450 nm was used as the reference wavelength). Lipid peroxidation was expressed as the MDA content in $\mu \mathrm{M}$ per $\mathrm{mg}$ of fresh weight.

\subsection{Immunohistochemistry}

To demonstrate the expression and localization of ionized calcium binding adaptor molecule 1 (Iba-1) and insulin-like growth factor 1 (IGF-1), immunohistochemistry was used as described previously. Coronal sections were deparaffinized with xylene and a series of graded alcohol solutions. Then, phosphate buffered saline solution (PBS) was used to wash the section.).Treated with citrate buffered solution, digested with 10 $\mu \mathrm{g} / \mathrm{ml}$ proteinase K-based solution, then blocked with $50 \mu \mathrm{l}$ normal goat serum, and reacted with anti-Iba- 1 $(1: 100)$ and anti-IGF-1 $(1: 100)$ overnight at $4^{\circ} \mathrm{C}$. Then washed off the primary antibodies and incubated with the corresponding secondary antibodies for $2 \mathrm{~h}$ at $37^{\circ} \mathrm{C}$, stained with $3,3^{\prime}$-diaminobenzidine (DAB), dehydrated with graded alcohol solution and mounted with triglyceride, visualized with a microscope (Eclipse50i, Nikon).

\subsection{Statistical Analysis}

All results were represented as the mean \pm standard deviation (SD). SPSS (SPSS Inc., Chicago, IL, USA) were used for further statistical analysis. Data were analyzed by one-way analysis of variance (ANOVA) when comparing mice in the same strain or by two-way ANOVA when comparing mice between different strains of corresponding age. Tukey's procedure was used for all post hoc tests.

\section{Results}

\subsection{K-PS Increased the Cortical Cross-sectional Area of Point C in SAMP10 Mice}

As shown in Figure 1A, black line circled up the measured cortical cross-sectional area of point $C$ in SAM mice of each group. The bar graph of Figure 1B shows the effect of K-PS on the cortical cross-sectional area at point C. Compared with the group of baseline P10 mice and R1 mice, the cortical cross-sectional area at point $\mathrm{C}$ in P10 mice had a $10.9 \%$ and $8.26 \%$ loss to a level of $9.4 \pm 0.688 \mathrm{~mm}^{2}(P<0.01$ and $P<0.05)$. In contrast, the cortical cross-sectional area at point $\mathrm{C}$ in mice treated with $\mathrm{K}-\mathrm{PS}_{\text {low }}$ showed a significant increase to a level of $10.29 \pm 0.52 \mathrm{~mm}^{2}$ compared with the P10 mice $(P<0.05)$. Figure $1 \mathrm{C}$ shows the location of point $\mathrm{C}$. 

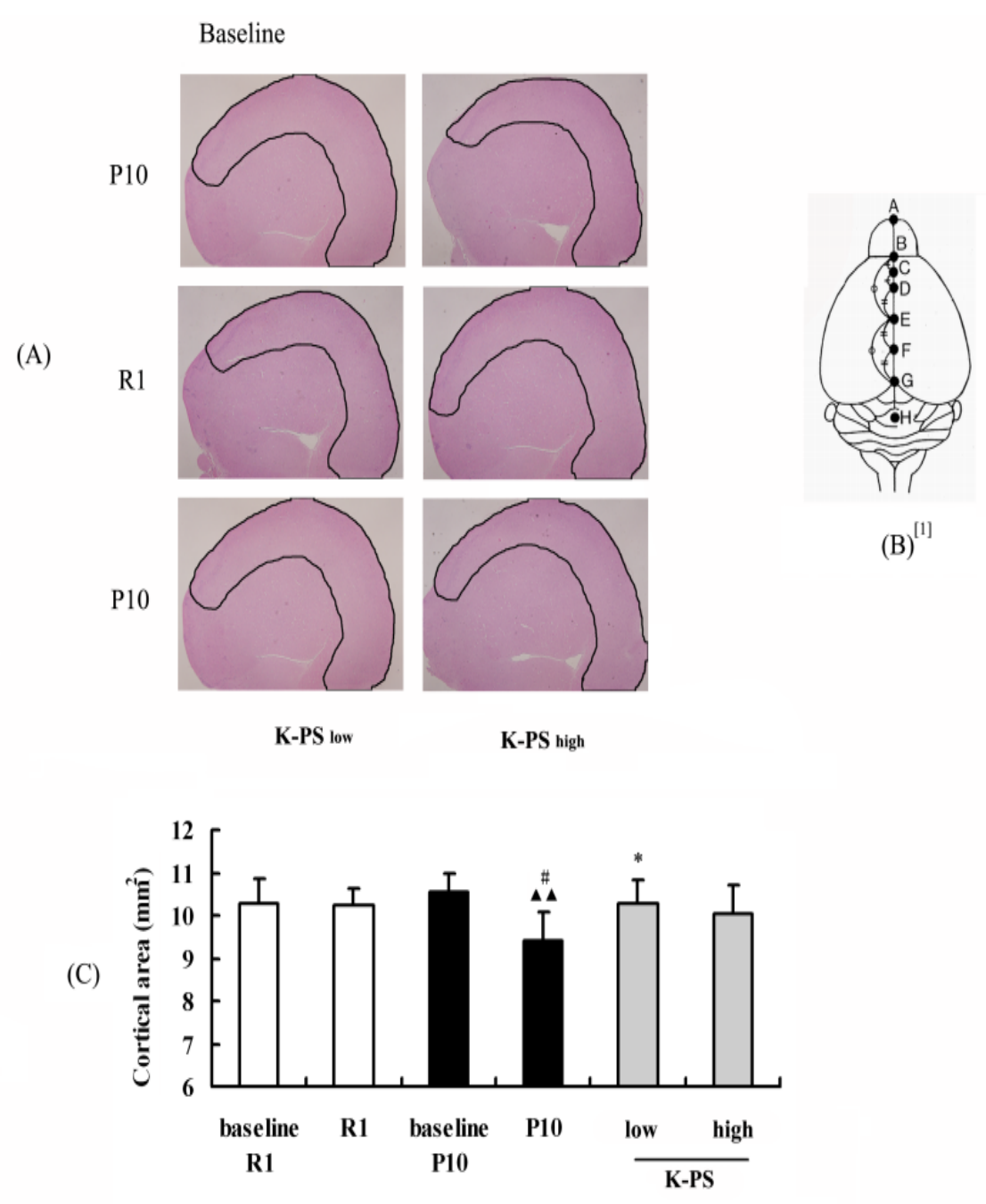

Figure 1. (A) Photo image showed brain slice with hematoxylin-eosin staining of SAM mice brains in each group. The part circled up by black line indicated the measurement of the cortical cross-sectional area. (B) Localization of atrophy-prone landmark pointed at a dorsal view of the brain in the photo image ${ }^{[1]}$. (C) Bar graphs show area changes in cross-sectional area of the neocortex in Plane C. Data expressed as mean \pm SD for

4-5 mice in each group. $\boldsymbol{\Delta} \boldsymbol{\Delta} P<0.01$ compared to the same month age in the SAMR1 strain. $\# P<0.05$ compared to the previous month age in the same strain. $* P<0.05$ compared to the P10.

\subsection{K-PS Increased the Nissl Body Number at Point C in SAMP10 Mice}

For the Nissl body count, we used thionien staining as described previously to observe changes at the cross-sectional area of the neocortex at point C. In baseline SAM mice and SAMR1 mice, the closely arranged neuron morphology was intact, and deep blue staining of lumpy or granular Nissl bodies could be seen in the neuronal cytoplasm. In P10 mice, Nissl bodies partially or entirely dissolved or disappeared and the neurons, with broken morphology, were arranged loosely. The number of deep blue staining of lumpy or granular in the K-PS ${ }_{\text {low }}$ and K-PS high groups was significantly greater than the P10 group (Figure 2A). As Figure 2B shows, the number of Nissl bodies in the P10 group declined to $33.83 \pm 7.6$ per high power field, with a $36.7 \%$ loss $(P<$ $0.01)$ compared with the baseline $\mathrm{P} 10$ group and a $32.3 \%$ loss $(P<0.01)$ compared with the R1 group. Conversely, the number of Nissl bodies in mice dosed with K-PS ${ }_{\text {low }}$ and K-PS ${ }_{\text {high }}$ was significantly higher than in the mice in group $\mathrm{P} 10(P=0.04$ and $P=0.03$ respectively $)$. 
(A)

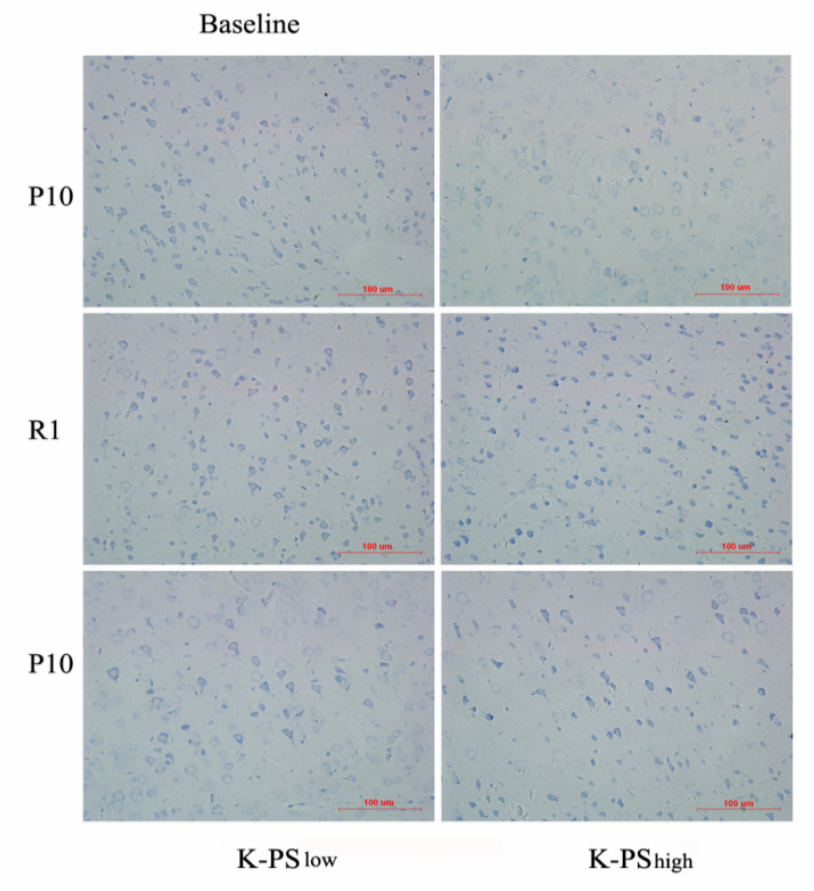

(B)

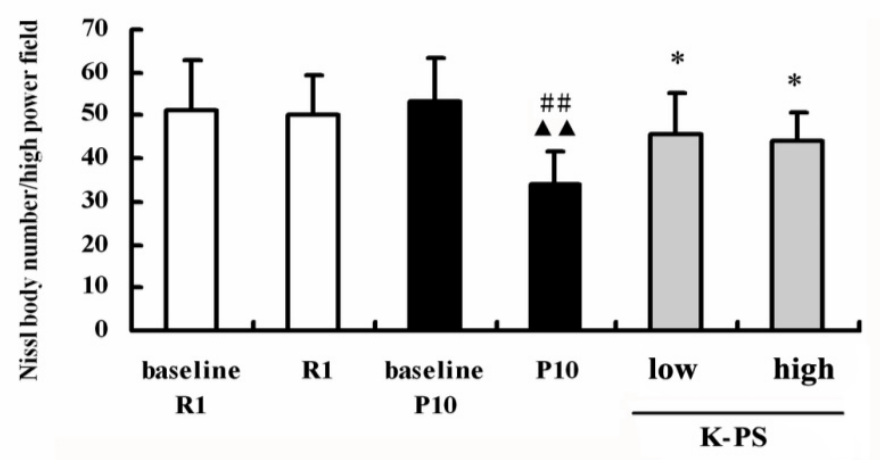

Figure 2. (A) Staining for Nissl bodies in the cross-sectional area of the neocortex at point C. In baseline SAM mice and SAMR1 mice, closely arranged neuron morphology was intact, and deep blue staining of lumpy or granular Nissl bodies could be seen in the neuronal cytoplasm. In P10 mice, Nissl bodies partially or entirely dissolved or disappeared and the neurons, with broken morphology, were arranged loosely. The deep blue staining of lumpy or granular Nissl bodies of neurons in the groups dosed with K-PS showed a significant increase compared with the P10 mice. (B) Bar graphs showed the count of Nissl bodies in the cross-section of the neocortex in Plane $\mathrm{C}$ in each group. $\boldsymbol{\Delta} \boldsymbol{\Delta} P<0.01$ compared to the same month-age in the SAMR1 strain. \#\# $P<0.01$ compared to the previous age in months in the same strain. ${ }^{*} P<0.05$ compared to the P10 mice. Scale bar $=100 \mu \mathrm{m}$.

\subsection{K-PS Improved Memory in SAMP10 Mice}

To determine the effect of K-PS on the ability to remember in SAM mice, we examined passive avoidance behavior using the step-down test. We observed that for memory, no remarkable difference was found in latency between the two strains of mice in the baseline groups. Compared with the baseline P10 mice and R1 mice, the latency of mice in group P10 was markedly shortened to $16.2 \pm 3.37 \mathrm{sec}(P<0.01)$. The latency of SAMP10 mice in the K-PS ${ }_{\text {low }}$ treated group was longer, with a remarkable increase compared with the mice in the P10 group $(P<0.05)$ (Figure 3A). In terms of error, the mice in group P10 showed a greater tendency to err compared with mice in the baseline P10 group $(P=0.1)$. Although the difference in error times was not statistically significant, K-PS appeared to reduce the tendency of error in P10 mice (Figure 3B). 
(A)

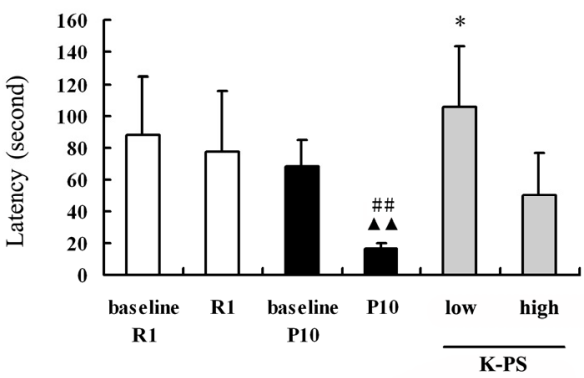

(B)

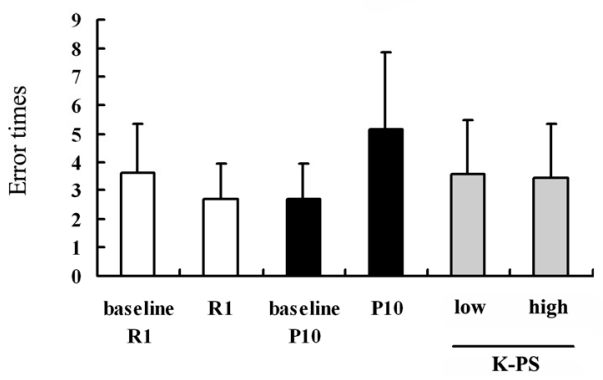

Figure 3. Changes in memory impairment via the step-down test: (A) Changes in the latency in SAMP10 mice. The mice administrated with $10 \mathrm{mg} \mathrm{kg}^{-1} \mathrm{~d}^{-1}$ for 75 days had a significantly longer latency compared to the P10 mice. (B) Changes in error times in SAMP10 mice. $\boldsymbol{\Delta} \boldsymbol{\Delta} P<0.01$ compared to the same month age in the SAMR1 strain. \#\# $P<0.01$ compared to the previous month age in the same strain. ${ }^{*} P<0.05$ compared to the P10.

\subsection{K-PS Increased GSH-Px Activities and Decreased MDA Content in SAMP10 Mice}

To establish the degree of oxidative stress in brain tissue, we examined GSH-Px activities and MDA levels in SAM mice. As Figure 4A showed, a significant difference in GSH-Px activities was found between the baseline P10, R1 and P10 groups $(P<0.01)$. Compared with the P10 group, a low or high dose of K-PS increased GSH-Px activities in SAMP10 mice markedly in a dose-dependent manner $(P<0.01$ or $P<0.001)$. MDA concentrations in SAM mice are presented in Figure 4B. Compared with the mice in the baseline P10 group, MDA levels in the K-PS dosed groups deceased remarkably $(P<0.01$ or $P<0.001)$.

(A)

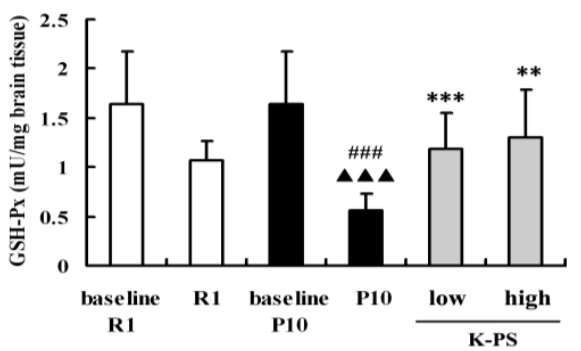

(B)

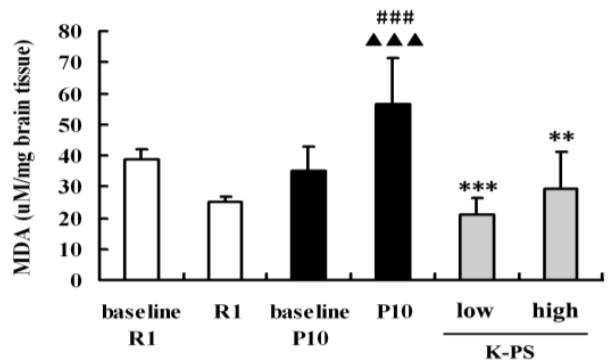

Figure 4. Changes in oxidative stress parameters: (A) GSH-Px activity in SAMP10 was markedly reduced compared with the controls. K-PS increased GSH-Px activity significantly in a dose-dependent manner. (B) P10 mice showed an increase in MDA concentrations compared with the controls. Both doses of K-PS deceased MDA concentrations in SAMP10 mice markedly. Data expressed as mean \pm SD for 4-6 mice in each group.

$\Delta \Delta \Delta P<0.001$ compared to the same month age in the SAMR1 strain. \#\#\# $P<0.001$ compared to the previous age in months in the same strain, $* * P<0.01, * * * P<0.001$ compared to the $\mathrm{P} 10$. 


\subsection{K-PS Downregulated Iba-1 Expression and Upregulated IGF-1 Expression in SAMP10 Mice}

Immunohistochemistry using Iba-1 antibodies showed an increase in immunoreactivity in P10 mice compared with baseline P10 mice and R1 mice, which indicated a strong microglial activity. The staining of Iba-1 was restricted within cytoplasm and stained as a brown dot. We found decreased expression of positive staining as detected by Iba-1 antibody in the cross-sectional area of the neocortex at point $\mathrm{C}$ in K-PS ${ }_{\text {high }}$ dosed mice compared to P10 mice (Figure 5). As shown in Figure 6, immunoreactivity of IGF-1 in P10 mice was remarkably downregulated compared with the baseline P10 and R1 groups. Compared with the P10 mice, the

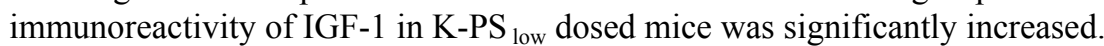

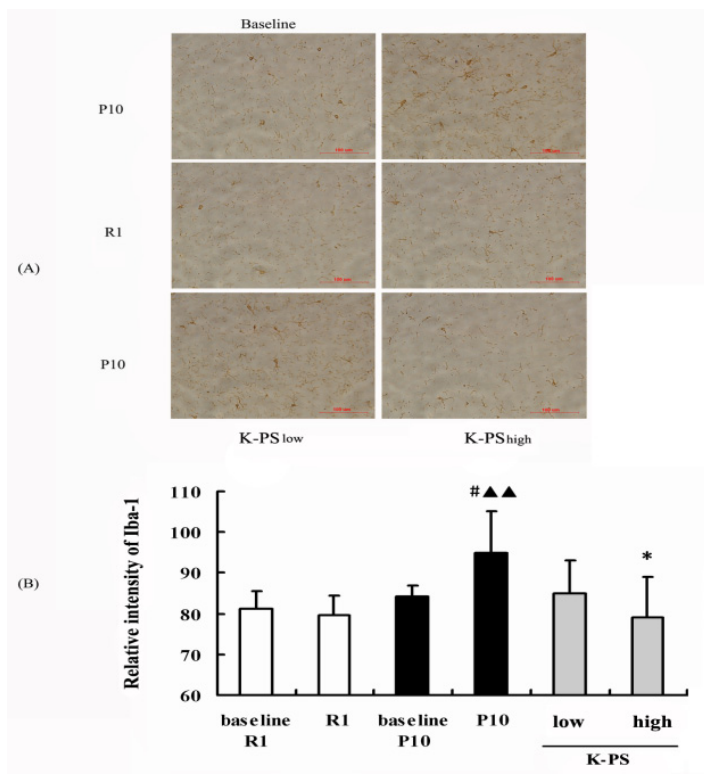

Figure 5. Immunohistochemical staining for Iba-1 in the cross-sectional area of the neocortex at point $\mathrm{C}$ in representative sections. (A) The positive-staining was confined to the microglial cytoplasm and appeared as brown granules with several branched processes. The intensity of Iba-1 immunoreactivity of P10 was increased compared with controls. After treatment by K-PS of $100 \mathrm{mg} \mathrm{kg}^{-1} \mathrm{~d}^{-1}$, Iba-1-positive cells were more weakly stained. (B) Bar graph shows the relative intensity of Iba-1. Scale bar $=100 \mu \mathrm{m}$.

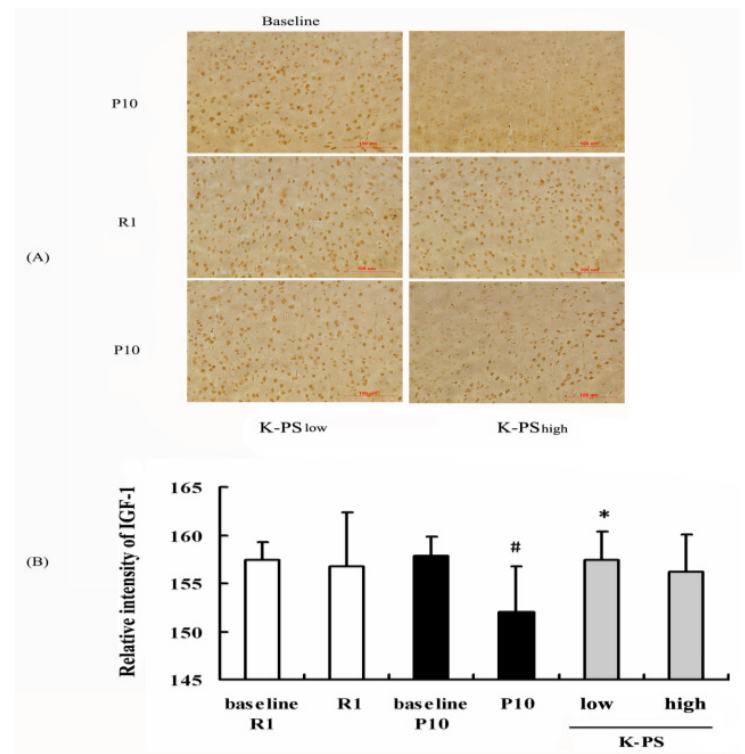

Figure 6. Immunohistochemical staining for IGF-1 in the cross-sectional area of the neocortex at point $\mathrm{C}$ in representative sections. (A) The positive-staining was confined to the cytoplasm and appeared as brown granules. The intensity of IGF-1 immunoreactivity of P10 was reduced compared with controls. After treatment by K-PS of $10 \mathrm{mg} \mathrm{kg}^{-1} \mathrm{~d}^{-1}$, IGF-1-positive cells were more strongly stained. (B) Bar graph shows the relative intensity of

IGF-1. Scale bar $=100 \mu \mathrm{m}$. 


\section{Discussion}

In the present study, we analyzed the anti-ageing effect of oral administration of K-PS. The results of this study showed an anti-ageing effect of K-PS on SAMP10 mice, reflected in less cortical atrophy, improved neurocognitive function, and suppression of Iba-1 expression as well as upregulation of IGF-1 expression. The SAMP10 strain of mice with age-related cerebral atrophy has been regarded as a model of age-associated neurodegeneration (Shimada et al., 2006). SAMP10 mice also experience an age-related loss of synapses and age-related dendritic retractions in prefrontal neurons. Atrophy is most exhibited at the anterior part of the cerebral cortex and olfactory structures. In this study, we examined the effect of K-PS on the cross-sectional area and Nissl body number in the frontal brain, the most prominent atrophy area. The cortical cross-sectional area in P10 mice was lower than that in R1 or baseline P10 mice during breeding. K-PS treatment with doses of 10 or $100 \mathrm{mg} \mathrm{kg}^{-1} \mathrm{~d}^{-1}$ increased the cortical cross-sectional area in SAMP10 mice, showing a similar effect to that on the number of Nissl bodies. The administration of a low or high dose of K-PS appears to improve the number of Nissl bodies indicating a recovery in neurons.

More and more evidence suggests that omega-3 HUFAs maintain and enhance the functions of the central nervous system (CNS), including learning and memory. The formation of newly-generated neurons in the hippocampus is associated with learn and memory abilities (Song et al., 2002; Schmidt-Hieber et al., 2004). When subjected to DHA chronically, both the young and aged rats' learning ability is improved (Gamoh et al., 1999; 2001). In addition, the neurogenesis of the granule cell layer in rats can be promoted by DHA (Kawakita et al., 2006). Furthermore, the dietary intake of both DHA-rich egg phospholipids and tuna oil can increase the maximum acetylcholine release from the hippocampus in the rats by increasing the DHA content. Evidence also indicates that EPA treatment can reduce the impairment of memory function from ageing (Okabe et al., 2011; Sinn et al., 2011). A longer latency was found in SAMP10 mice with low doses of K-PS, which suggests that accelerated senescence can be suppressed by the administration of K-PS.

Recently, oxidative stress has been regarded as an important causal factor of senescence (Toru et al., 2010). The components of K-PS (DHA or EPA) have been found to have antioxidative effects on cells and animals (Jensen et al., 2009; Kim et al., 2007; Pan et al., 2009). As an important lipid peroxidation product, MDA can be considered as an indicator for the oxidative damage extend. Abnormal alteration of MDA content is related to memory impairment, as reported in previous studies (Um et al., 2006). GSH is a sensitive indicator of the ability of a cell or tissue to resist damage. When oxygen free radicals increase, cells and tissues need more nicotinamide adenine dinucleotide phosphate (NADP) to be recovered from the glutathione cycle, where glutathione is reduced to GSH. The damaged GSH system may cause oxidative stress and destruction of the nigrostriatal pathway as well as rendering the pathway susceptible to a toxic insult (Jha et al., 2000). Our results show that the SAMP10 mice suffered from oxidative stress more than SAMR1 mice due to the inherited cerebral atrophy, both low and high doses of K-PS can increase GSH-Px activity and decrease MDA levels significantly. This suggests that K-PS treatment offers antioxidant benefits. As oxidative stress within mitochondria has been regarded as a major risk factor of ageing (Romano et al., 2010), we assume that the antioxidative properties of K-PS may play a role in this anti-ageing effect on SAMP10 mice.

Microglial cells are involved in infection, hypoxic-ischemic brain injury disease and degenerative diseases of the nervous system in the pathogenesis (Moon et al., 2009; Wang et al., 2011). According to the former studies about microglial cells morphology in age-related disease, researchers have observed that microglia become activated in aged rats (Ogura et al., 1994; Kawamata et al., 1998). Quantitative analysis has shown that abnormalities of microglia in the early stage may cause the SAMP10 mice vulnerable to age-related neuronal degeneration (Hasegawa-Ishii et al., 2011). Iba-1, a marker of microglial activation (Ahmed et al., 2007), was evaluated in this paper; the results showed that K-PS ${ }_{\text {low }}$ significantly downregulated the expression of Iba-1 which indicated that activated microglial cells were inhibited to a certain extent. A previous study indicated that hydrogen peroxide originated from the dismutation of superoxide produced by nicotinamide adenine dinucleotide phosphate (NADPH) oxidase may regulate the microglial cells change (Mander et al., 2006). NADPH oxidase is the source of reactive oxygen species (ROS) generated by activated microglial cells (Hosokawa 2002; Chiba et al., 2005). Thus, we assume that the inhibitory effect of K-PS on microglial cells may be related to the antioxidant effect on SAMP10 mice as discussed above.

Insulin-like growth factor 1 (IGF-1) is an endocrine hormone primarily synthesized in the liver. The IGF-1/IGF-1R system is thought to exert beneficial effects on the function of neurons in the central nervous system, including antioxidant effects (Yusuke et al., 2010) and anti-apoptotic effects (Peruzzi, et al., 1999), preventing the loss of neurons (Guan et al., 1999). The results of our study show that K-PS low upregulated the expression of IGF-1 in the cortex, which suggests that K-PS could prevent the diminishing of IGF-1. In addition, 
the loss of IGF which is necessary for learning and memory may cause cognitive disabilities (Lupien et al., 2003). The molecular mechanisms of signaling via cross-talk between IGF-1 and ROS-mediated redox signaling pathways may play an important role in regulation of ageing and longevity (John, 2009). Consequently, the improvement in memory and the antioxidant effect may be partially due to the upregulation of IGF-1 by K-PS.

KPS contained 11.3\% EPA and 5.6\% DHA. The high dose had 10-times more EPA or DHA than those of the low dose. Based on the above results, we can see that low dose had almost the same effect with the high dose on the mice and show no dose-dependent effect. A few factors that may have contributed to our detecting of no significant difference between low and high dose include the following: (1) Low dose of K-PS may basically occupy the role of targets and show a saturated pharmacological effects so that high dose of K-PS can't play better the pharmacological effects. (2) It is important to note that the efficacy of K-PS is partly due to the multi-component, which allows for a comprehensive regulatory effect. Compared with single-component receptor drugs, each component in K-PS plays a different role in the overall pharmacological action, which makes it more difficult to define the dose-effect relationship. (3) High dose of PS may generate pharmacological effects other than what we have done. Taken together, we plan to consider a more sensitive dose evaluation of K-PS to reveal a benefit of anti-brain ageing effect in future studies.

\section{Conclusion}

Taken together, our findings suggest that a 10 or $100 \mathrm{mg} \mathrm{kg}^{-1} \mathrm{~d}^{-1}$ dose of K-PS may reduce oxidative damage and subsequent cortical atrophy as well as Nissl body loss, resulting in improved memory ability. These protective effects may be due to K-PS's inhibition of microglial activation and upregulation of IGF-1. Thus, K-PS may be useful in preventing or slowing progression of cortical atrophy and neuronal damage induced by ageing, and K-PS may become an important source of nutrition in the future.

\section{Acknowledgements}

This work was supported by grants from the National Science \& Technology Major Project of China (No.2009ZX09502-014), the Ministry of Education and Key Laboratory of Chinese Internal Medicine, and the National Natural Science Foundation of China (No. 81102679).

\section{References}

Ahmed, Z., Shaw, G., Sharma, V. P., Yang, C., McGowan, E., \& Dickson, D. W. (2007). Actin-binding proteins coronin-1a and IBA-1 are effective microglial markers for immunohistochemistry. J. Histochem. Cytochem., 55, 687-700. http://dx.doi.org/ 10.1369/jhc.6A7156.2007

Amaducci, L. (1988). Phosphatidylserine in the treatment of Alzheimer's disease: results of a multicenter study. Psychopharmacol. Bull., 24, 130-134.

Blokland, A., Honig, W., Brouns, F., \& Jolles, J. (1999). Cognition-enhancing properties of subchronic phosphatidylserine (PS) treatment in middle-aged rats: comparison of bovine cortex PS with egg PS and soybean PS. Nutrition, 15, 778-783.

Chiba, Y., Yamashita, Y., Ueno, M., Fujisawa, H., Hirayoshi, K., Hohmura, K., ... Hosokawa, M. (2005). Cultured murine dermal fibroblast-like cells from senescence-accelerated mice as in vitro models for higher oxidative stress due to mitochondrial alterations. J. Gerontol. Ser. A. Biol. Sci. Med. Sci., 60, 1087-1098. http://dx.doi.org/10.1093/gerona/60.9.1087

Corwin, J., Dean, R. L., Bartus, R. T., Rotrosen, J., \& Watkins, D. L. (1985). Behavioral effects of phosphatidylserine in the aged Fisher 344 rat: amelioration of passive avoidance deficits without changes in $\begin{array}{lllll}\text { psychomotor task performance. Neurobiol. } & \text { Aging, }\end{array}$ http://dx.doi.org/10.1016/0197-4580(85)90065-X

Crook, T. H., Tinklenberg, J., Yesavage, J., Petrie, W., Nunzi, M. G., \& Massari, D. C. (1991). Effects of phophatidylserine in age-associated memory impairment. Neurology, 41, 644-649. http://dx.doi.org/10.1212/WNL.41.5.644

Delwaide, P. J., Gyselynck-Mambourg, A. M., Hurlet, A., \& Ylieff, M. (1986). Double-blind randomized controlled study of phosphatidylserine in senile demented patients. Acta. Neurol. Scand., 73, 136-140. http://dx.doi.org/10.1111/j.1600-0404

Gamoh, S., Hashimoto, M., Hossain, S., \& Masumura, S. (2001). Chronic administration of docosahexaenoic acid improves the performance of radial arm maze task in aged rats. Clin. Exp. Pharmacol. Physiol., 28, 266-270. http://dx.doi.org/10.1046/j.1440-1681.2001.03437.x 
Gamoh, S., Hashimoto, M., Sugioka, K., Shahdat Hossain, M., Hata, N., Misawa, Y., \& Masumura, S. (1999). Chronic administration of docosahexaenoic acid improves reference memory-related learning ability in young rats. Neoroscience, 93, 237-241. http://dx.doi.org/10.1016/S0306-4522(99)00107-4

Guan, J., Waldvogel, H. J., Faull, R. L., Gluckman, P. D., \& Williams, C. E. (1999). The effects of the N-terminal tripeptide of insulin-like growth factor-1, glycine-proline-glutamate in different regions following hypoxic-ischemic brain injury in adult rats. Neuroscience, 89, 649-659. http://dx.doi.org/10.1016/S0306-4522(98)00338-8

Hasegawa-Ishii, S. Takei, S., Chiba, Y., Furukawa, A., Umegaki, H., Iguchi, A., ... Shimada, A. (2011). Morphological impairments in microglia precede age-related neuronal degeneration in $\begin{array}{llll}\text { senescence-accelerated mice. } & \text { Neuropathology, } & 31, & 20-28 \text {. }\end{array}$ http://dx.doi.org/10.1111/j.1440-1789.2010.01126.x

Higashi, Y., Sukhanov, S., Anwar, A., Shai, S. Y., \& Delafontaine, P. (2010). IGF-1, oxidative stress, and atheroprotection. Trends. Endocrinol. Metab., 21, 245-254. http://dx.doi.org/10.1016/j.tem.2009.12.005

Hosokawa, M. (2002). A higher oxidative status accelerates senescence and aggravates age-dependent disorders in SAMP strains of mice. Mech. Ageing. Dev., 123, 1553-1561. http://dx.doi.org/10.1016/S0047-6374(02)00091-X

Jensen, I. J., Abrahamsen, H., Maehre, H. K., \& Elvevoll, E. O. (2009). Changes in antioxidative capacity of saithe (Pollachius virens) and shrimp (Pandalus borealis) during in vitro digestion. J. Agric. Food. Chem., 57, 10928-10932. http://dx.doi.org/10.1021/jf9023849

Jha, N., Jurma, O., Lalli, G., Liu, Y., Pettus, E. H., Greenamyre, J. T., ... Andersen, J. K. (2000). Glutathione depletion in PC12 results in selective inhibition of mitochondrial complex I activity. Implications for Parkinson's disease. J. Biol. Chem., 275, 26096-26101. http://dx.doi.org/10.1074/jbc.M000120200

John, P. (2009). Insulin/IGF-1 and ROS signaling pathway cross-talk in aging and longevity deterlowation. Mol. Cell. Endocrinol., 299, 89-100. http://dx.doi.org/10.1016/j.mce.2008.11.025

Jorissen, B. L., Brouns, F., Van Boxtel, M. P., Ponds, R. W., Verhey, F. R., Jolles, J., \& Riedel, W. J. (2001). The influence of soy-derived phosphatidylserine on cognition in age-associated memory impairment. Nutr. Neurosci., 4, 121-134.

Kawakita, E., Hashimoto, M., \& Shido, O. (2006). Docosahexaenoic acid promotes neurogenesis in vitro and in vivo. Neuroscience, 139, 991-997. http://dx.doi.org/10.1016/j.neuroscience.2006.01.021

Kawamata, T., Akiguchi, I., Maeda, K., Tanaka, C., Higuchi, K., Hosokawa, M., \& Takeda. T. (1998). Age-related changes in the brains of senescence-accelerated mice (SAM): association with glial and endothelial reactions. Microsc. Res. Tech., http://dx.doi.org/10.1002/(SICI)1097-0029(19981001)43:1<59::AID-JEMT9>3.0.CO;2-X

Kim, Y. J., \& Chung, H. Y. (2007). Antioxidative and anti-inflammatory actions of docosahexaenoic acid and eicosapentaenoic acid in renal epithelial cells and macrophages. J. Med. Food., 10, $225-231$. http://dx.doi.org/10.1089/jmf.2006.092

Lawrence, R. A., \& Burk, R. F. (1976). Glutathione peroxidase activity in selenium-deficient rat liver. Biochem. Biophys. Res. Commun., 71, 952-958.

Lee, B., Sur, B. J., Han, J. J., Shim, I., Her, S., Lee, H. J., \& Hahm, D. H. (2010). Krill phosphatidylserine improves learning and memory in Morris water maze in aged rats. Prog. Neuropsychopharmacol. Biol. Psychiatry, 34, 1085-1093. http://dx.doi.org/10.1016/j.pnpbp.2010.05.031

Lupien, S. B., Bluhm, E. J., \& Ishii, D. N. (2003). Systemic insulin-like growth factor-I adlowistration prevents cognitive impairment in diabetic rats, and brain IGF regulates learning/memory in normal adult rats. $J$. Neurosci. Res., 74, 512-523. http://dx.doi.org/10.1002/jnr.10791

Mander, P. K., Jekabsone, A., \& Brown, G. C. (2006). Microglia proliferation is regulated by hydrogen peroxide from NADPH oxidase. J. Immunol., 176, 1046-1052.

Moon, J. B., Lee, C. H., Park, C. W., Cho, J. H., Hwang, I. K., Yoo, K. Y., ... Won, M. H. (2009). Neuronal degeneration and microglial activation in the ischemic dentate gyrus of the gerbil. J. Vet. Med. Sci., 71, 159-166. http://dx.doi.org/10.1292/jvms.001381

Morrison, J. H., \& Hof, P. R. (2007). Life and death of neurons in the aging cerebral cortex. Int. Rev. Neurobiol., 81, 41-57. http://dx.doi.org/10.1016/S0074-7742(06)81004-4 
Ogura, K., Ogawa, M., \& Yoshida, M. (1994). Effects of ageing on microglia in the normal rat brain: immunohistochemical observations. Neuroreport, 5, 1224-1226.

Okabe, N., Nakamura, T., Toyoshima, T., Miyamoto, O., Lu, F., \& Itano. T. (2011). Eicosapentaenoic acid prevents memory impairment after ischemia by inhibiting inflammatory response and oxidative damage. $J$. Stroke. Cerebrovasc. Dis., 20, 188-195. http://dx.doi.org/10.1016/j.jstrokecerebrovasdis.2009.11.016

Pan, H. C., Kao, T. K., Ou, Y. C., Yang, D.Y., Yen, Y. J., Wang, C. C., ... Chen, C. J. (2009). Protective effect of docosahexaenoic acid against brain injury in ischemic rats. J. Nutr. Biochem., 20, 715-725. http://dx.doi.org/10.1016/j.jnutbio.2008.06.014

Peruzzi, F., Prisco, M., Dews, M., Salomoni, P., Grassilli, E., Romano, G., Calabretta, B., \& Baserga, R. (1999). Multiple signaling pathways of the insulin-like growth factor 1 receptor in protection from apoptosis. Mol. Cell. Biol., 19, 7203-7215.

Prasad, S., Sung B., \& Aggarwal, B. B. (2012). Age-associated chronic diseases require age-old medicine: Role of chronic inflammation. Prev. Med., 54, Suppl: S29-S37. http://dx.doi.org/10.1016/j.ypmed.2011.11.011

Raz, N., Rodrigue, K. M., Head, D., Kennedy, K. M., \& Acker, J. D. (2004). Differential aging of the medial temporal lobe: a study of a five-year change. Neurology, 62, 433-438. http://dx.doi.org/10.1212/01.WNL.0000106466.09835.46

Romano, A. D., Serviddio, G., de Matthaeis, A., Bellanti, F., \& Vendemiale, G. (2010). Oxidative stress and aging. J. Nephrol., 23, S29-S36.

Sasaki, T., Unno, K., Tahara, S., \& Kaneko, T. (2010). Age-related increase of reactive oxygen generation in the brains of mammals and birds: Is reactive oxygen a signaling molecule to deterlowe the ageing process and life span? Geriatr. Gerontol. Int., 10, S10-S24. http://dx.doi.org/10.1111/j.1447-0594.2010.00601.x

Schmidt-Hieber, C., Jonas, P., \& Bischofberger, J. (2004). Enhanced synaptic plasticity in newly generated granule cells of the adult hippocampus. Nature, 429, 184-187. http://dx.doi.org/10.1038/nature02553

Shimada, A. (1999). Age-dependent, inherited cerebral atrophy and cognitive dysfunction in SAMP10 mice. Neurobiol. Aging, 20, 125-536. http://dx.doi.org/10.1016/S0197-4580(99)00044-5

Shimada, A., Hosokawa, M., Ohta, A., Akiguchi, I., \& Takeda, T. (1994). Localization of atrophy-prone areas in the ageing mouse brain: comparison between the brain atrophy model SAM-P/10 and the normal control SAM-R/1. Neuroscience, 59, 859-569. http://dx.doi.org/10.1016/0306-4522(94)90290-9

Shimada, A., Keino, H., Satoh, M., Kishikawa, M., \& Hosokawa, M. (2003). Age-related loss of synapses in the frontal cortex of SAMP10 mouse: a model of cerebral degeneration. Synapse, 48, 198-204. http://dx.doi.org/10.1002/syn.10209

Shimada, A., Ohta, A., Akiguchi, I., \& Takeda, T. (1992). Inbred SAM-P/10 as a mouse model of spontaneous, inherited brain atrophy. J. Neuropathol. Exp. Neurol., 51, 440-450.

Shimada, A., Tsuzuki, M., Keino, H., Satoh, M., Chiba, Y., Saitoh, Y., \& Hosokawa, M. (2006). Apical vulnerability to dendritic retraction in prefrontal neurones of ageing SAMP10 mouse: a model of cerebral degeneration. Neuropathol. Appl. Neurobiol., 32, 1-14. http://dx.doi.org/10.1111/j.1365-2990.2006.00632.x

Shuchang, H., Qiao, N., Piye, N., Mingwei, H., Xiaoshu, S., Feng, S., Sheng, W., \& Opler, M. (2008). Protective effects of gastrodia elata on aluminium-chloride-induced learning impairments and alterations of amino acid neurotransmitter release in adult rats. Restor. Neurol. Neurosci., 26, 467-573.

Simić, G., Kostović, I., Winblad ,B., \& Bogdanović, N. (1997). Volume and number of neurons of the human hippocampal formation in normal aging and Alzheimer's disease. J. Comp. Neurol., 379, 482-494.

Sinn, N., Milte, C. M., Street, S. J., Buckley, J. D., Coates, A. M., Petkov, J., \& Howe, P. R. (2011). Effects of n-3 fatty acids, EPA v. DHA, on depressive symptoms, quality of life, memory and executive function in older adults with mild cognitive impairment: a 6-month randomised controlled trial. Br. J. Nutr., 20, 1-12. http://dx.doi.org/10.1017/S0007114511004788

Song, H. J., Stevens, C. F., \& Gage, F. H. (2002). Neural stem cells from adult hippocampus develop essential properties of functional CNS neurons. Nat. Neurosci, 5, 438-445. http://dx.doi.org/10.1038/nn844

Stern, Y., Mayeux, R., Sano, M., Hauser, W. A., \& Bush, T. (1987). Predictors of disease course in patients with probable Alzheimer's disease. Neurology, 37, 1649-1653. http://dx.doi.org/10.1212/WNL.37.10.1649

Svennerholm, L., Boström, K., Helander, C. G., \& Jungbjer, B. (1991). Membrane lipids in the aging human 
brain. J. Neurochem., 56, 2051-2059. http://dx.doi.org/10.1111/j.1471-4159.1991.tb03466.x

Um, M. Y., Choi, W. H., Aan, J. Y., Kim, S. R., \& Ha, T. Y. (2006). Protective effect of Polygonum multiflorum Thunb on amyloid $\beta$-peptide 25-35 induced cognitive deficits in mice. J. Ethnopharmacoloy, 104, 144-148. http://dx.doi.org/10.1016/j.jep.2005.08.054

Vakhapova, V., Cohen, T., Richter, Y., Herzog, Y., \& Korczyn, A. D. (2010). Phosphatidylserine containing omega-3 fatty acids may improve memory abilities in non-demented elderly with memory complaints: a double-blind placebo-controlled trial. Dement. Geriatr. Cogn. Disord., 29, 467-474. http://dx.doi.org/10.1159/000310330

Vance, J. E., \& Steenbergen, R. (2005). Metabolism and functions of phosphatidylserine. Prog. Lipid. Res., 44, 207-324. http://dx.doi.org/10.1016/j.plipres.2005.05.001

Wang, J., Li, P. T., Du, H., Hou, J. C., Li, W. H., Pan, Y. S., Hua, Q., \& Chen, H. C. (2011). Impact of paracrine signals from brain microvascular endothelial cells on microglial proliferation and migration. Brain. Res. Bull., 86, 53-59. http://dx.doi.org/10.1016/j.brainresbull.2011.06.016

Zanotti, A., Aporti, F., Rubini, R., \& Toffano, G. (1986). Learning and memory deficits in rats during aging: effect of phosphatidylserine treatments. (p 323). Padova : Liviana.

Zhang, H., Jiang, Y., He, Z.\& Ma, M. (2005). Cadmium accumulation and oxidative burst in garlic (Allium sativum). J. Plant. Physiol., 162, 977-984. http://dx.doi.org/10.1016/j.jplph.2004.10.001 